Reply

\title{
Reply to Comment on Choi et al. Correlation between Ionospheric TEC and the DCB Stability of GNSS Receivers from 2014 to 2016. Remote Sens. 2019, 11, 2657
}

\author{
Byung-Kyu Choi ${ }^{1, * \mathbb{D}}$, Dong-Hyo Sohn ${ }^{1}$ and Sang Jeong Lee ${ }^{2} \mathbb{D}$ \\ 1 Space Science Division, Korea Astronomy and Space Science Institute, Daejeon 34055, Korea; \\ dhsohn@kasi.re.kr \\ 2 Department of Electronics Engineering, Chungnam National University, Daejeon 34134, Korea; \\ eesjl@cnu.ac.kr \\ * Correspondence: bkchoi@kasi.re.kr; Tel.: +82-42-865-3237
}

Received: 9 October 2020; Accepted: 22 October 2020; Published: 26 October 2020

\begin{abstract}
Choi et al. (2019) suggested that ionospheric total electron content (TEC) and receiver differential code bias (rDCB) stability have a strong correlation during a period of two years from 2014 to 2016. This article is a response to Zhong et al. (2020), who pointed out that the long-term variations of the GPS DCBs are mainly attributed to the satellite replacement rather than the ionospheric variability. In this issue, we investigated the center for orbit determination in Europe (CODE) Global Ionosphere Maps (GIM) products from 2000 to 2020. In this study, changes in TEC and receiver DCB (rDCB) root mean squares (RMS) at Bogota (BOGT) station still have a clear correlation. In addition, there was a moderate correlation between satellite DCB RMS and rDCB RMS. As a result, we suggest that rDCB can be affected simultaneously by GPS SDCB as well as ionospheric activity.
\end{abstract}

Keywords: receiver DCB; ionospheric TEC; satellite DCB; root mean squares (RMS)

The Global Navigation Satellite System (GNSS) Differential Code Biases (DCB) is the largest error source in estimating the total electron content (TEC) of the ionosphere. Many studies have reported that DCB is affected by a variety of factors [1-6]. There can be significant jumps [7] and other features in DCB related to the replacement of the receiver and antenna.

Choi et al. [8] showed that ionospheric TEC and receiver DCB (rDCB) root mean squares (RMS) have a strong correlation during a period of two years from 2014 to 2016. In addition, they focused on the relation between $\mathrm{rDCB}$ stability and ionospheric activity rather than $\mathrm{rDCB}$ itself. However, Zhong et al. [9] pointed out that the long-term variations of the GPS DCBs are mainly attributed to the satellite replacement rather than the ionospheric variability. Interestingly, they also focused on the GPS DCB changes and did not mention the relationship between rDCB stability and ionospheric activity. As suggested by Zhong et al. [9], rDCB and satellite DCB may have a significant correlation.

In this issue, Choi et al. [8] suggested that changes in the rDCB RMS still have continuity after the replacement of a receiver. Replacing hardware such as GNSS receivers or satellites can seriously affect the DCB. However, large changes in DCB with hardware replacement may be usually instantaneous.

For more reliable analysis of the results presented by Choi et al. [8], we investigated the center for orbit determination in Europe (CODE) Global Ionosphere Maps (GIM) data from 2000 to 2020. Figure 1 shows the time series of vertical TEC units (VTECU), rDCB, and rDCB RMS (Stability) at BOGT station, respectively. A band-pass filter was applied to isolate specific changes for GIM-TEC and rDCB RMS. As shown in Figure 1a, the variations of VTEC are closely related to solar activities. 
They also show an exact annual and semi-annual cycle. To analyze the relationship between VTEC and rDCB RMS during this period, we calculated band-pass filtered $(0 \sim 0.05 \mathrm{~Hz}) \mathrm{rDCB}$ RMS plotted in Figure $1 \mathrm{a}$ as a red solid line. In CODE's GIM products, the rDCB RMS values of the BOGT station exist only from 2003 to 2020. Filtered rDCB RMS showed annual and semi-annual patterns similar to GIM-TEC. However, there were no regular changes in rDCB RMS from 2006 to 2011, and solar activity was relatively weak during this period.
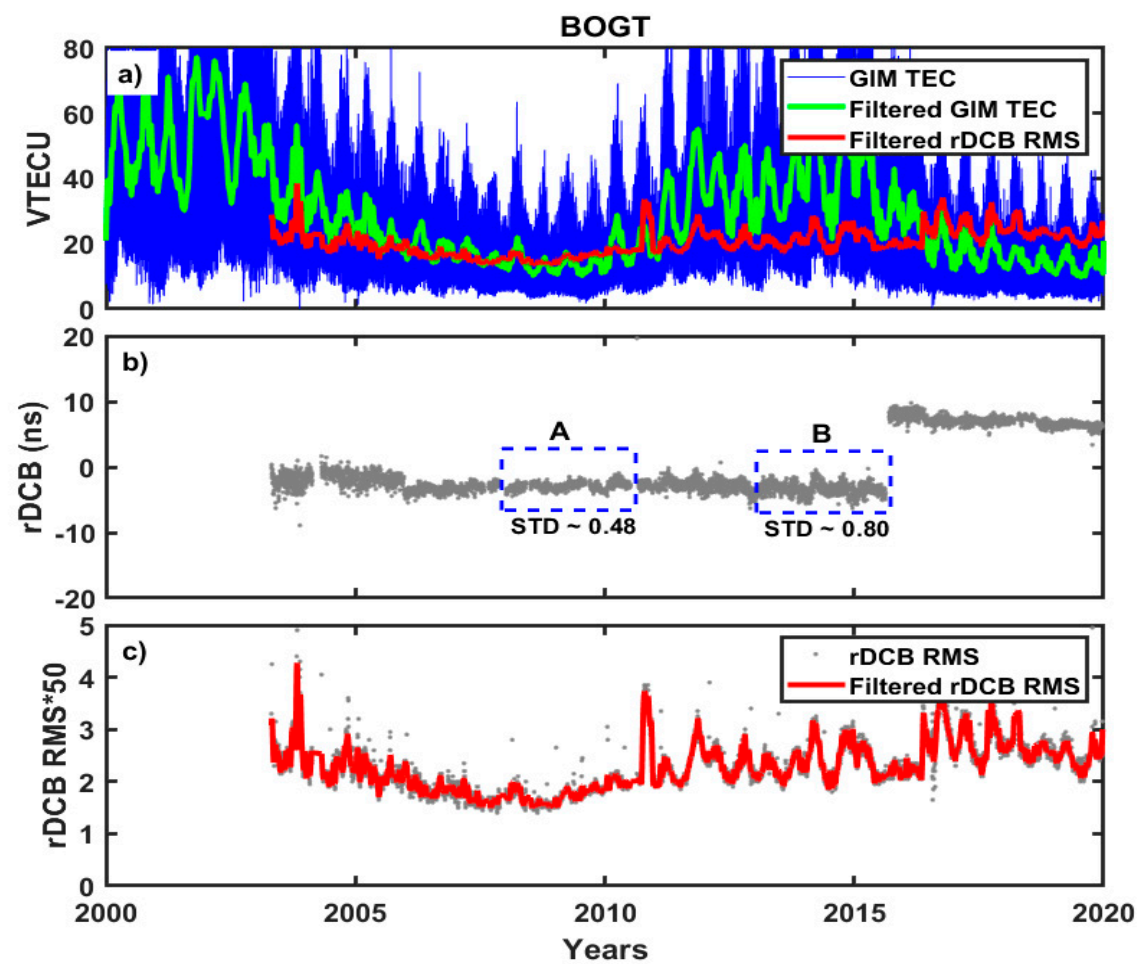

Figure 1. (a) Time series of vertical total electron content (VTEC), band-pass filtered (0 0.05 Hz) VTEC, and band-pass filtered $(0 \sim 0.05 \mathrm{~Hz})$ receiver differential code bias (rDCB) route mean squares (RMS) at BOGT station $\left(4.64^{\circ} \mathrm{N}, 74.08^{\circ} \mathrm{W}\right)$ from 2000 to 2020 . The blue and green solid lines indicate the variations of Global Ionosphere Maps (GIM)-TEC and band-pass filtered GIM-TEC, respectively. The red solid line indicates band-pass filtered rDCB RMS, taken from the bottom panel c). (b) Time series of GPS rDCB values from 2003 to 2020 . The blue dashed rectangles " $\mathrm{A}$ " and " $\mathrm{B}$ " show the changes of $\mathrm{rDCB}$ with ionospheric activity. (c) Time series of rDCB RMS at BOGT station. The grey dots and red solid line denote the raw rDCB RMS and band-pass filtered $(0 \sim 0.05 \mathrm{~Hz})$ rDCB RMS, respectively.

Nevertheless, changes in VTEC and rDCB RMS appear to have a clear correlation. Figure $1 \mathrm{~b}$ shows the time series of GPS rDCB at BOGT station $\left(4.64^{\circ} \mathrm{N}, 74.08^{\circ} \mathrm{W}\right)$ from about 2003 to 2020 . A significant jump in rDCB was detected due to receiver replacement. Changes in rDCB do not show any regular patterns similar to VTEC. Zhong et al. [9] suggested that the variations of rDCB are not caused by the ionospheric variability, which may be consistent with our results. The blue dashed rectangles " $\mathrm{A}$ " and "B" in Figure $1 \mathrm{~b}$ represent the changes of $\mathrm{rDCB}$ in low- and high-ionospheric activity, respectively. The standard deviations (STD) of $\mathrm{rDCB}$ in the two sections " $\mathrm{A}$ " and " $\mathrm{B}$ " were about 0.48 and 0.80 , respectively. Variations in $\mathrm{rDCB}$ can see to be unstable during high ionospheric activity. On the other hand, they are stable when ionospheric activity is relatively low. Therefore, rDCB changes can be associated with ionospheric activity. In addition, as shown in Figure 1c, rDCB RMS values were small and stable during low-ionospheric activity.

Figure 2 presents the time series of GPS satellite P1-P2 DCB (sDCB), band-pass filtered (0 1.5Hz) sDCB, and rDCB RMS from 2000 to 2020, respectively. Changes in DCB for all GPS satellites are shown in Figure 2a. In Figure 2a, it is not easy to distinguish specific correlations with ionospheric activity in 
GNSS sDCB changes. To analyze the relationship between sDCB and rDCB, we considered the most stable satellites with no PRN changes [7,10]. As plotted in Figure 2a, GPS PRN 28 was selected.
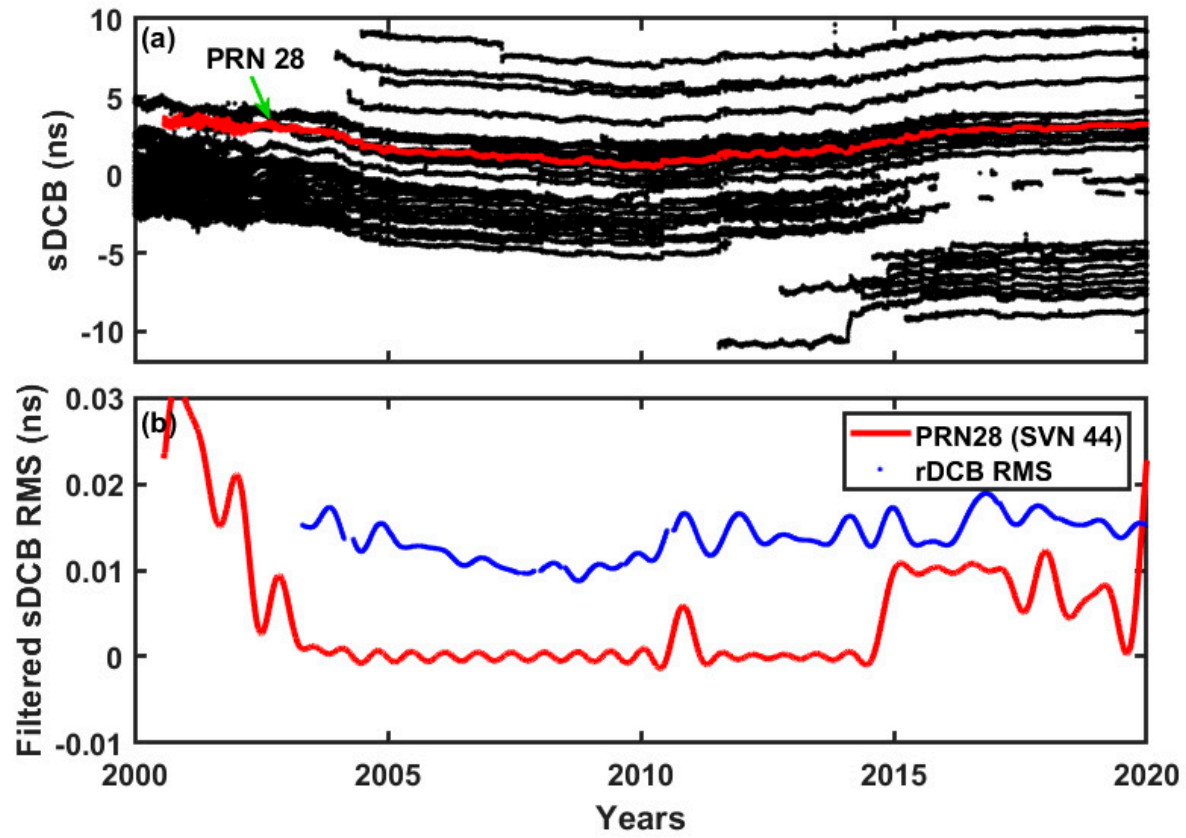

Figure 2. (a) Time series of all GPS satellite P1-P2 DCB (sDCB) from 2000 to 2020. The red dots indicate the changes in DCB values for GPS satellite PRN 28. (b) Time series of band-pass filtered $(0 \sim 1.5 \mathrm{~Hz})$ sDCB RMS for GPS PRN 28 (SVN 44), and band-pass filtered (0 1.5 Hz) rDCB RMS from 2000 to 2020. The red solid line and blue dots represent the filtered RMS changes for GPS satellite PRN 28 DCB and $\mathrm{rDCB}$ at BOGT station, respectively.

Zhong et al. [9] also reported that the ionospheric activity does not cause the long-term variations of the GPS sDCB. However, sDCBs have relatively large RMS values for solar maximum from about 2000 to 2003, as shown in Figure 2b. In addition, the filtered sDCB RMS values for GPS PRN 28 show very similar periodic patterns. These cycles can be coincident with a period of a draconitic year of GPS satellites suggested by Zhong et al. [9]. Furthermore, there was a significant jump in changes in GPS PRN 28 sDCB RMS in 2015. In early 2015, CODE changed a solar radiation pressure (SRP) model from the old empirical CODE orbit model (ECOM) to the new ECOM2 for GNSS orbit determination [11]. A change in the CODE SRP model can cause DCB variations. For the relationship between sDCB RMS and rDCB RMS, we plotted the band-filtered rDCB RMS as the blue dots in Figure $2 b$. There is some correlation between the two variables. The correlation coefficient $(R)$ between the two variables was about 0.58 . It indicates that there is a moderate correlation between sDCB RMS and rDCB RMS.

Choi et al. [8] suggested that the correlation between TEC and rDCB RMS shows the apparent latitudinal dependency with ionosphere variability. It can be related to TEC distributions. Zhang et al. [12] reported that the accuracy of $\mathrm{rDCB}$ estimation is dependent on the receiver's location. The $\mathrm{rDCB}$ estimation accuracy can be lower in low-latitude regions because TEC distributions at low latitudes are much more complicated than those at mid and high latitudes [13,14]. Strugarek et al. [15] reported that the GNSS signal is more affected during the periods of the high ionospheric activity, which causes problems with precise orbit determination of low Earth orbit satellites. What we emphasize is that it can be difficult to specifically explain the latitudinal dependency of rDCB with changes in GPS sDCB only.

In general, the global ionospheric TEC is modeled by the spherical harmonic function. To separate the satellite DCBs and rDCBs in ionospheric GPS-TEC estimation, we consider a constraint that the sum of all GPS satellite DCBs becomes zero. Under this condition, variables such as TEC, satellite DCBs, and rDCBs have to be calculated simultaneously. Each of the estimated variables can be influenced by 
each other. As suggested by Zhong et al. [9], there was a clear correlation between sDCB and rDCB. However, we suggest that variables are not estimated as an independent variable but are correlated. Therefore, $\mathrm{rDCB}$ can be affected simultaneously by GPS sDCB as well as ionospheric activity.

Author Contributions: Methodology, B.-K.C.; software, B.-K.C.; validation, B.-K.C. and D.-H.S.; investigation, B.-K.C.; writing-original draft preparation, B.-K.C.; writing-review and editing, B.-K.C., D.-H.S. and S.J.L.; visualization, B.-K.C. and D.-H.S. All authors have read and agreed to the published version of the manuscript.

Funding: This research received no external funding.

Acknowledgments: The authors would like to thank the IGS for providing GNSS data and IONEX GIM products. This study was supported by the 2020 Primary Project of the Korea Astronomy and Space Science Institute. The authors would also like to take the opportunity to thank the editor and the anonymous reviewers for their constructive comments.

Conflicts of Interest: The authors declare no conflict of interest.

\section{References}

1. Sardón, E.; Zarraoa, N. Estimation of Total Electron Content Using GPS Data: How Stable Are the Differential Satellite and Receiver Instrumental Biases. Radio Sci. 1997, 32, 1899-1910. [CrossRef]

2. Zhang, W.; Zhang, D.H.; Xiao, Z. The influence of geomagnetic storms on the estimation of GPS instrumental biases. Ann. Geophys. 2009, 27, 1613-1623. [CrossRef]

3. Coster, A.; Williams, J.; Weatherwax, A.; Rideout, W.; Herne, D. Accuracy of GPS total electron content: GPS receiver bias temperature dependence. Radio Sci. 2013, 48, 190-196. [CrossRef]

4. Zhang, D.; Shi, H.; Jin, Y.; Zhang, W.; Hao, Y.; Xiao, Z. The variation of the estimated GPS instrumental bias and its possible connection with ionospheric variability. Sci. China Tech. Sci. 2014, 57, 67-79. [CrossRef]

5. Themens, D.R.; Jayachandran, P.T.; Langley, R.B. The nature of GPS differential receiver bias variability: An examination in the polar cap region. J. Geophys. Res. Space Phys. 2015, 120, 8155-8175. [CrossRef]

6. Choi, B.-K.; Lee, S.J. The influence of grounding on GPS receiver differential code biases. ASR 2018, 62, 457-463. [CrossRef]

7. Xiang, Y.; Xu, Z.; Gao, Y.; Yu, W. Understanding long-term variations in GPS differential code biases. GPS Solut. 2020, 24, 118. [CrossRef]

8. Choi, B.-K.; Sohn, D.-H.; Lee, S.J. Correlation between Ionospheric TEC and the DCB Stability of GNSS Receiver from 2014 to 2016. Remote Sens. 2019, 11, 2657. [CrossRef]

9. Zhong, J.; Lei, J.; Yue, X. Comment on Choi et al. Correlation between Ionospheric TEC and the DCB Stability of GNSS Receiver from 2014 to 2016. Remote Sens. 2019, 11, 2657. Remote Sens. 2020, 12, 3496. [CrossRef]

10. Villiger, A.; Schaer, S.; Dach, R.; Prange, L.; Sušnik, A.; Jäggi, A. Determination of GNSS pseudo-absolute code biases and their long-term combination. J. Geod. 2019, 93, 1487-1500. [CrossRef]

11. Arnold, D.; Meindl, M.; Beutler, G.; Dach, R.; Schaer, S.; Lutz, S.; Prange, L.; Sośnica, K.; Mervart, L.; Jäggi, A. CODE's new solar radiation pressure model for GNSS orbit determination. J. Geod. 2015, 89, 775-791. [CrossRef]

12. Zhang, D.H.; Zhang, W.; Li, Q.; Shi, L.Q.; Xiao, Z. Accuracy Analysis of the GPS Instrumental Bias Estimated from Observations in Middle and Low Latitudes. Ann. Geophys. 2010, 28, 1571-1580. [CrossRef]

13. Brunini, C.; Azpilicueta, F. GPS Slant Total Electron Content Accuracy Using the Single Layer Model Under Different Geomagnetic Regions and Ionospheric Conditions. J. Geod. 2010, 84, 293-304. [CrossRef]

14. Kao, S.; Chen, W.; Weng, D.; Ji, S. Factors affecting the estimation of GPS receiver instrumental biases. Survey Rev. 2013, 45, 59-67. [CrossRef]

15. Strugarek, D.; Sośnica, K.; Jäggi, A. Characteristics of GOCE orbits based on Satellite Laser Ranging. Adv. Spa. Res. 2019, 63, 417-431. [CrossRef]

Publisher's Note: MDPI stays neutral with regard to jurisdictional claims in published maps and institutional affiliations. 